\title{
Peierls Condition and Number of Ground States
}

\author{
W. Holsztynski ${ }^{1}$ and J. Slawny ${ }^{2 \star}$ \\ ${ }^{1}$ Mathematics Department, The University of Western Ontario, London, Canada \\ ${ }^{2}$ Institut de Physique Théorique, Université de Louvain, B-Louvain, Belgium
}

\begin{abstract}
Recently Pirogov and Sinai developped a theory of phase transitions in systems satisfying Peierls condition. We give a criterium for the Peierls condition to hold and apply it to several systems. In particular we prove that ferromagnetic system satisfies the Peierls condition iff its (internal) symmetry group is finite. And using an algebraic argument we show that in two dimensions the symmetry groups of reduced translation invariant systems is finite.
\end{abstract}

\section{Introduction}

A theory of phase transitions at low temperatures in general classical lattice systems was developped recently by Pirogov and Sinai $[8,9]$. It applies to systems satisfying the Peierls condition of $[8,4,9]$. In particular, finitness of the number of (periodic) ground states is assumed.

The main results of this paper are: Criterium for Peierls condition to hold (Section 3), a necessary and sufficient condition for ferromagnetic systems to satisfy Peierls condition (Sections 10 and 11) and a theorem on finitness of the number of ground states of ferromagnetic systems in two dimensions (Section 12). In addition, to demonstrate how the Criterium works, we apply it to several models. The criterium applies to all models we consider and often yields more complete picture than previously obtained.

In Sections 1 and 2 we introduce the framework and recall the Peierls condition. In Section 3 we formulate and prove Criterium; in the proof we take advantage of the flexibility of the Gerzik-Debrushin $[5,4,8,9]$ definition of contours which allows us to replace previous considerations based on special symmetries of models by a general compactness argument. After preparatory remarks of Section 4, in Sections 5-9 we apply Criterium to several models; the discussion of the antiferromagnet in Section 5 which leads to well known results of Dobrushin [3] is especially detailed.

* Present address: Department of Mathematics, Rutgers University, New Brunswick, NJ 08903, USA 
Applying Criterium to ferromagnets (Sections 10, 11) we obtain that a ferromagnetic model satisfies Peierls condition if and only it its (internal) symmetry group $\mathscr{S}$ is finite. On the other hand in our work $[7,6,11]$ on the number of phases in ferromagnetic systems at low temperatures Peierls condition is not assumed and we treat cases with infinite number of ground states; [7] will contain a more detailed comparison of the results.

Exploiting algebraic properties of ferromagnetic systems we prove (Section 12), that any two-dimensional spin $\frac{1}{2}$ ferromagnetic system which is $\mathbb{Z}^{2}$-invariant (not only periodic) has a version ("reduced version") with a finite number of ground states, which thus satisfies Peierls condition.

We need more algebra to show that if $\mathscr{S}$ is infinite then it containes an infinite number of periodic elements-result we use in Section 10. In Appendix the problem is reduced to a question in commutative algebra which has a positive solution.

In Section 13 we discuss systems with constrains.

In all the examples we consider the Peierls condition breaks down because for some values of the parameters an infinite number of ground states occurs. But as examples discussed in Section 14 show, infinitude of ground states may lead to a variety of situations at non-zero temperatures. These seems to exist no general theory describing it.

\section{Framework}

As in [10] except that we allow for constrains; see $[9,14]$.

Let $S$ be a finite set and let $\mathscr{X}$ be a closed subspace of $S^{\mathbb{L}}$, where $\mathbb{L}=\mathbb{Z}^{v}$ for simplicity. For $\Lambda \subset \mathbb{L}, \mathrm{pr}_{\Lambda}$ is the projection $S^{\mathbb{L}} \rightarrow S^{\Lambda}$ and $\mathrm{pr}_{\Lambda}^{\prime}=\mathrm{pr}_{\mathbb{L} \backslash \Lambda} ; \mathscr{X}_{\Lambda}=\mathrm{pr}_{\Lambda} \mathscr{X}$.

A potential $\Phi$ is a collection $\left(\Phi_{A}\right)$ where $\Phi_{A}$ is a real valued function on $\mathscr{X}_{\Lambda}, \Lambda \subset \mathbb{L} . \Phi_{\Lambda}$ will be identified with the function $\Phi_{A}{ }^{\circ} \mathrm{pr}_{A}$ on $\mathscr{X}$. We consider only the case of periodic systems, i.e. when there exists a subgroup of $\mathbb{Z}^{v}$ of a finite index leaving invariant $\mathscr{X}$ and $\Phi$. Moreover $\Phi$ is a assumed to have finite range $\left(\Phi_{\Lambda}\right.$ is zero if the diameter of $\Lambda$ is large enough).

Two configurations $X, Y \in \mathscr{X}$ are equal at infinity, $X \sim Y$, if there exists finite $\Lambda \subset \mathbb{L}$ such that $\operatorname{pr}_{\Lambda}^{\prime} X=\operatorname{pr}_{\Lambda}^{\prime} Y$. The (relative) Hamiltonian $H=H_{\Phi}$ is defined by

$$
H(X \mid Y)=\sum_{\Lambda}\left(\Phi_{\Lambda}(X)-\Phi_{\Lambda}(Y)\right) \text { for } \quad X \sim Y
$$

we write sometimes

$$
H=\sum_{\Lambda} \Phi_{\Lambda}
$$

Two potentials are equivalent if they yield the same relative Hamiltonian.

\section{Ground States and Peierls Condition [9]}

Let $\mathscr{X}^{\text {per }}$ be the family of periodic elements of $\mathscr{X}^{1}, X \in \mathscr{X}^{\text {per }}$ is a ground state (of $H$ ) if

$$
H(X \mid Y) \leqq 0 \quad \text { for any } \quad Y \sim X
$$

$1 \quad$ An element of $\mathscr{X}$ is periodic if it is invariant under a subgroup of $\mathbb{Z}^{v}$ of a finite index. Similarly are defined periodic elements of any $\mathbb{Z}^{v}$-space 
$\mathscr{G}$ or $\mathscr{G}(H)$ is the family of all ground states of the Hamiltonian $H$.

We will assume that the following lemma holds.

Lemma. Let for $X \in \mathscr{X}^{\text {per }}$

$$
e_{\Phi}(X)=\lim _{\Lambda} \frac{1}{|\Lambda|} \sum_{\Lambda^{\prime} \subset \Lambda} \Phi_{\Lambda^{\prime}}(X) .
$$

Then $X$ is a ground state for $H_{\Phi}$ iff

$$
e_{\Phi}(X) \leqq e_{\Phi}(Y), \quad \forall Y \in \mathscr{X}^{\text {per }} .
$$

This is proved to hold for systems without constrains, $\mathscr{X}=S^{\mathbb{L}}$, in [9]. It is not hard to find an example of a system with constrains for which the lemma does not hold.

For a natural number $N$ and $a \in \mathbb{L}$ let

$N(a)=\{x \in \mathbb{L}:|a-x| \leqq N\}$,

and let the $N$-boundary of a configuration $X \in \mathscr{X}$ be

$$
B_{N}(X)=\cup\left\{N(a): \operatorname{pr}_{N(a)} X \notin \operatorname{pr}_{N(a)} \mathscr{G}\right\} .
$$

Definition. $\quad H$ satisfieds Peierls condition if $\mathscr{G}(H)$ is finite and for each $N$ large enough there exists $\varrho>0$ such that if $X \sim Y, Y \in \mathscr{G}$,

$$
H(X \mid Y) \geqq \varrho \operatorname{card}\left(B_{N}(X)\right) .
$$

This differs slightly from the definition of [9] but is easily seen to be equivalent to it.

\section{Criterium}

With the preceding notation and assumptions let

$$
\phi_{\Lambda}=\min _{X \in \mathscr{X}} \Phi_{\Lambda}(X)
$$

and

$\mathscr{G}^{\prime}=\left\{X \in \mathscr{X}: \Phi_{\Lambda}(X)=\phi_{\Lambda}, \forall \Lambda\right\}$.

If $\mathscr{G}^{\prime \text { per }}$ is non-empty then

$\mathscr{G}\left(H_{\Phi}\right)=\mathscr{G}^{\prime \text { per }}$;

if $\mathscr{G}^{\prime}$ is finite then $\mathscr{G}^{\prime}=\mathscr{G}\left(H_{\Phi}\right)$ and the Peierls condition holds for $H_{\Phi}$.

Proof of Criterium. That in case $\mathscr{G}^{\text {per }}$ is non-empty it coincides with $\mathscr{G}\left(H_{\Phi}\right)$ follows at once from Lemma 2.

Any translation that leaves $\Phi$ invariant maps $\mathscr{G}^{\prime}$ into itself. If $\mathscr{G}^{\prime}$ is finite then the subgroup of the invariance group of $\Phi$ which leaves $\mathscr{G}^{\prime}$ pointwise invariant is of a finite index, and therefore of a finite index in $\mathbb{Z}^{\nu}$. Thus the configurations of $\mathscr{G}^{\prime}$ are periodic and therefore $\mathscr{G}^{\prime}=\mathscr{G}\left(H_{\Phi}\right)$. It remains to show that finitness of $\mathscr{G}^{\prime}$ implies Peierls condition. 
Let $\mathscr{G}_{A}=\operatorname{pr}_{\Lambda} \mathscr{G}$, and consider any finite $\tilde{\Lambda} \subset \mathbb{L}$; there exists $r>0$ such that for any $X \in \mathscr{X}_{\tilde{\Lambda}} \backslash \mathscr{G}_{\tilde{\Lambda}}$ and any $Y \in \mathscr{X}$ with $\operatorname{pr}_{\tilde{\Lambda}} Y=X$ there is $\Lambda$ such that

$$
\operatorname{dist}(\tilde{\Lambda}, \Lambda) \leqq r, \Phi_{\Lambda}(Y) \neq \phi_{\Lambda} .
$$

Otherwise there would exist sequences $\left(X_{n}\right) \subset \mathscr{X}_{\tilde{\Lambda}} \backslash^{\mathscr{G}_{\tilde{A}}}$ and $\left(Y_{n}\right) \subset \mathscr{X}$ such that $\operatorname{pr}_{\tilde{\Lambda}} Y_{n}=X_{n}, \Phi_{\Lambda}\left(Y_{n}\right)=\phi_{\Lambda}$ when $\operatorname{dist}(\tilde{\Lambda}, \Lambda) \leqq n$. Since $\mathscr{X}_{\tilde{\Lambda}}$ is finite we may assume $X_{n}=X, \forall n$, and since $\mathscr{X}$ is compact $\left(Y_{n}\right)$ has a limit point $Y$. But then $Y \in \mathscr{G}^{\prime}=\mathscr{G}\left(H_{\Phi}\right)$ and $\operatorname{pr}_{\tilde{\Lambda}} Y=X$ which contradicts the assumption that $X \notin \mathscr{G}_{\tilde{\Lambda}}$.

Let $N$ be a positive number; by periodicity and (3.1) there exists $r=r(N)$ such that if $\operatorname{pr}_{N(a)} X \in \mathscr{G}_{N(a)}, X \in \mathscr{X}$, then there is $\Lambda_{a}$, depending on $a$ but not on $X$, satisfying:

$$
\operatorname{dist}\left(N(a), \Lambda_{a}\right) \leqq r, \Phi_{\Lambda_{a}}(X) \neq \phi_{\Lambda_{a}} .
$$

If

$$
\operatorname{dist}\left(a, a^{\prime}\right)>\delta=2 N+2 r+\max \left\{\operatorname{diam} \Lambda: \Phi_{\Lambda} \neq \phi_{\Lambda}\right\}
$$

then $\Lambda_{a} \neq \Lambda_{a}$.

\section{Let}

$$
\varepsilon=\inf \left\{\Phi_{\Lambda}(X)-\phi_{\Lambda}: \Phi_{\Lambda}(X) \neq \phi_{\Lambda}, X \in \mathscr{X}, \Lambda \subset \mathbb{L}\right\} ;
$$

since $\Phi$ is periodic and finite range, $\varepsilon \neq 0$. Now for $X \sim Y, Y \in \mathscr{G}$, the relative energy

$$
H(X \mid Y)=\sum_{\Lambda}\left(\Phi_{\Lambda}(X)-\Phi_{\Lambda}(Y)\right)=\sum_{\Lambda}\left(\Phi_{\Lambda}(X)-\phi_{\Lambda}\right)
$$

is bounded from below by $\varepsilon$ times the cardinality of any subset $A$ of $\mathbb{L}$ such that $\operatorname{pr}_{N(a)} X \notin \mathscr{G}_{N(a)}, \operatorname{dist}(a, b)>\delta$, for any $a, b \in A, a \neq b$. Therefore

$$
H(X \mid Y) \geqq \varepsilon \times(2 N+1)^{-v} \times \delta^{-v} \times\left|B_{N}(X)\right| .
$$

The criterium is proved.

\section{Application of Criterium}

In applications one has to choose for given Hamiltonian the potential to which Criterium applies. This will be illustrated in the following discussion of a number of examples.

$X \in \mathscr{X}_{\Lambda}$ such that $\Phi_{\Lambda}(X)=\phi_{\Lambda}$ will be called a local ground state. Since $\Lambda$ 's overlap the problem is in first determining local ground states and then in patching them together into ground states.

Drawing Diagrams; the Closure Condition. Usually the lines (more generally the hypersurfaces) of the zero-temperature phase diagram divide the space of parameters into a number of open regions with Peierls condition satisfied in each of them. More precisely, in each case we have a finite dimensional space $\mathscr{H}$ of interactions and a family $\left(U_{i}\right)_{i \in I}$ of open subsets of $\mathscr{H}$ such that $\bigcup_{i} U_{i}$ is dense in $\mathscr{H}$ and any $H \in U_{i}$ has the same (finite) family $\mathscr{G}_{i}$ of ground states, $i \in I$. Certainly

$$
\mathscr{G}(H) \supset U\left\{\mathscr{G}_{i}: H \text { is in the dosure of } U_{i}\right\}
$$


but in general the inclusion is proper, i.e. at points where few of the $U_{i}$ 's meet there may appear new ground state which is in none of $\mathscr{G}_{i}$ 's. Most often this happens because at this meeting points we are allowed to combine local ground states coming from different $U_{i}$ 's (see Sections 5-9). Usually infinitude of ground states results then. We say that an interaction $H$ (or corresponding point of the diagram) satisfies closure condition if in $(*)$ we have equality in place of the inclusion.

The lines with infinite number of ground states are fattened on the diagrams.

\section{Antiferromagnet [3]}

We consider the two-dimensional case only; generalization to higher dimensions is obvious.

In examples of Sections $5-10, S=\{-1,1\}$, and for $a \in \mathbb{Z}^{v}, \sigma_{a}=\operatorname{pr}_{\{a\}}$, . Here

$$
H=2 \sum_{\langle a, b\rangle} \sigma_{a} \sigma_{b}-4 h \sum_{a} \sigma_{a}
$$

with $\sum_{\langle a, b\rangle}$ denoting sum over pairs of nearest neighbor points of the lattice. Let $a=0, b=e_{1}, c=e_{1}+e_{2}, d=e_{2}$, where $e_{i}$ is the canonical base of $\mathbb{Z}^{2}$, let

$$
\Phi_{0}=\sigma_{a} \sigma_{b}+\sigma_{b} \sigma_{c}+\sigma_{c} \sigma_{d}+\sigma_{a} \sigma_{d}-h\left(\sigma_{a}+\sigma_{b}+\sigma_{c}+\sigma_{d}\right)
$$

and let

$$
\begin{aligned}
\Phi_{\Lambda} & =\tau_{x} \Phi_{0} \quad \text { if } \quad \Lambda=\tau_{x}\left(\Lambda_{0}\right), \Lambda_{0}=\{a, b, c, d\} \\
& =0 \text { otherwise }
\end{aligned}
$$

where $\tau_{x}$ stays for translation by $x \in \mathbb{Z}^{v}$. Then $H=\sum_{\Lambda} \Phi_{\Lambda}$; we apply Criterium to $\Phi$.

Computation yields the following local ground states in $\Lambda_{0}$ :

$$
\begin{aligned}
& h>2\left(\begin{array}{ll}
+ & + \\
+ & +
\end{array}\right) \\
& h=2\left(\begin{array}{ll}
+ & + \\
+ & +
\end{array}\right)\left(\begin{array}{ll}
- & + \\
+ & -
\end{array}\right)\left(\begin{array}{ll}
+ & - \\
- & +
\end{array}\right)\left(\begin{array}{ll}
- & + \\
+ & +
\end{array}\right)\left(\begin{array}{ll}
+ & - \\
+ & +
\end{array}\right)\left(\begin{array}{ll}
+ & + \\
+ & -
\end{array}\right)\left(\begin{array}{ll}
+ & + \\
- & +
\end{array}\right) \\
& -2<h<2\left(\begin{array}{ll}
- & + \\
+ & -
\end{array}\right)\left(\begin{array}{ll}
+ & - \\
- & +
\end{array}\right) \\
& h=-2\left(\begin{array}{ll}
- & - \\
- & -
\end{array}\right)\left(\begin{array}{ll}
+ & - \\
- & +
\end{array}\right)\left(\begin{array}{ll}
- & + \\
+ & -
\end{array}\right)\left(\begin{array}{ll}
+ & - \\
- & -
\end{array}\right)\left(\begin{array}{ll}
- & + \\
- & -
\end{array}\right)\left(\begin{array}{ll}
- & - \\
- & +
\end{array}\right)\left(\begin{array}{ll}
- & - \\
+ & -
\end{array}\right) \\
& h<-2\left(\begin{array}{ll}
- & - \\
- & -
\end{array}\right)
\end{aligned}
$$

in a selfexplanatory notation. 
Since $\Phi$ is $\mathbb{Z}^{2}$-invariant, ground states are obtained by patching together these configurations, if such patching is at all possible. The following obtaines : for $|h|<2$ or $|h|>2$ each of the local ground states admits unique extension to a ground state; for $|h|=2$ the extension is not unique and one gets infinite number of ground states. This is because for $h=2$, for instance, the last four local ground states allow to pass from $\left(\begin{array}{ll}+ & + \\ + & +\end{array}\right)$ to $\left(\begin{array}{ll}- & + \\ + & -\end{array}\right)$ or $\left(\begin{array}{ll}+ & - \\ - & +\end{array}\right)$ as in

$$
\begin{aligned}
& ++++-+-+++ \\
& ++++-+-++++.
\end{aligned}
$$

\section{Antiferromagnet in a Staggered Magnetic Field}

$$
H=2 \sum_{n . n} \sigma_{a} \sigma_{b}-4 h \sum_{a} \sigma_{a}-4 \eta\left(\sum_{a \in \mathbb{L}_{0}} \sigma_{a}-\sum_{a \in \mathbb{L}_{1}} \sigma_{a}\right)
$$

where

$$
\mathbb{L}_{0}=\left\{\left(x_{1}, x_{2}\right) \in \mathbb{Z}^{2}: x_{1}+x_{2} \text { is even }\right\}, \quad \mathbb{L}_{1}=\mathbb{Z}^{2} \backslash \mathbb{L}_{0} .
$$

With the notation of Section 5 , let

$$
\begin{aligned}
\Phi_{\Lambda}= & \tau_{x}\left[\sigma_{a} \sigma_{b}+\sigma_{b} \sigma_{c}+\sigma_{c} \sigma_{d}+\sigma_{a} \sigma_{d}-h\left(\sigma_{a}+\sigma_{b}+\sigma_{c}+\sigma_{d}\right)\right] \\
& +\varepsilon(x) \eta \tau_{x}\left[\sigma_{a}+\sigma_{d}-\sigma_{b}-\sigma_{c}\right]
\end{aligned}
$$

if $\Lambda=\tau_{x} \Lambda_{0}$ and $\Phi_{\Lambda}=0$ otherwise; here $\varepsilon(x)=1$ if $x \in \mathbb{L}_{0}, \varepsilon(x)=-1$ if $x \in \mathbb{L}_{1}$. Then $H=H_{\Phi}$. One has to analyse two types of $\Phi_{\Lambda}$, say $\Phi_{\Lambda_{0}}$ and $\Phi_{\tau_{e_{1}}} \Lambda_{0}$. Proceeding like in Section 5 the following $T=0$ phase diagram results :

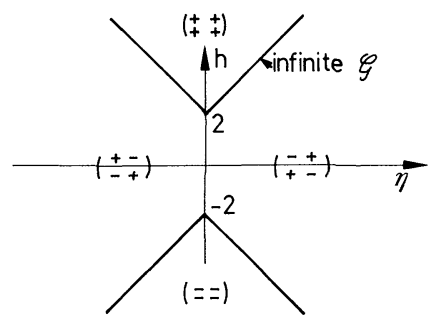

Fig. 1

The lines of the diagram divide the $(\eta, h)$-plane into four open regions with one ground state in each of them. There is an infinite number of ground states on the inclined half lines. All other points of the diagram satisfy Peierls condition and the closure condition of Section 4.

\section{The Preceding Model with a Next Nearest Neighbour Ferromagnetic Interaction in Addition}

(Fisher's antiferromagnet of [9] in a staggered magnetic field)

$$
H=H^{(6)}-J \sum_{n \cdot n \cdot n} \sigma_{a} \sigma_{b}, \quad J>0
$$


Defining the potential like in Section 6 we see that the only effect of the ferromagnetic next nearest neighbour interaction is an elimination of the local ground states

$$
\left(\begin{array}{ll}
- & + \\
+ & +
\end{array}\right),\left(\begin{array}{ll}
+ & - \\
+ & +
\end{array}\right) \text { etc. }
$$

Thus Criterium yields the following phase diagram at zero temperature:

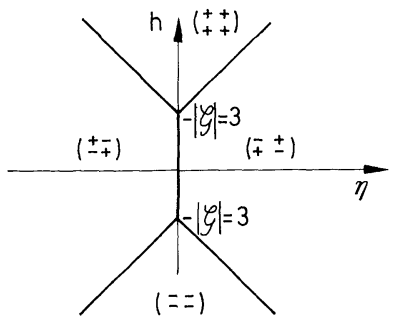

Fig. 2

with Peierls condition satisfied everywhere; the closure condition is satisfied at all points of the diagram.

This model of metamagnet is supposed to exhibit a tricritical behaviour with phase diagram like that of [13, Fig. 2]. The Pirogov-Sinai theory yields the low temperature portion of this phase diagram.

\section{Gertsik-Dobrushin Model [5]}

$$
H=\varepsilon \sum_{\langle a b\rangle} \sigma_{a} \sigma_{b}+J \sum_{n . n . n} \sigma_{a} \sigma_{b}+h \sum_{a} \sigma_{a} \quad a, b \in \mathbb{Z}^{v} .
$$

When $\varepsilon=0$ the model splits into two ferromagnetic $(J<0)$ or antiferromagnetic models $(J>0)$. Hence it is enough to consider two cases: $\varepsilon>0$ and $\varepsilon<0$.

In contradistinction to models considered so far, here the two-dimensional case is much simpler than the higher dimensional; we will comment on this later and now we consider only the $v=2$ case. Results obtained here are more complete than those in [5].

Decomposing $H$ as in preceding sections and applying Criterium we obtain the following zero-temperature phase diagrams.

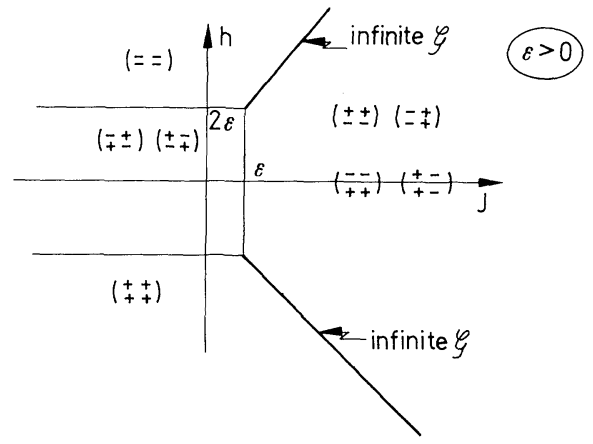

Fig. 3

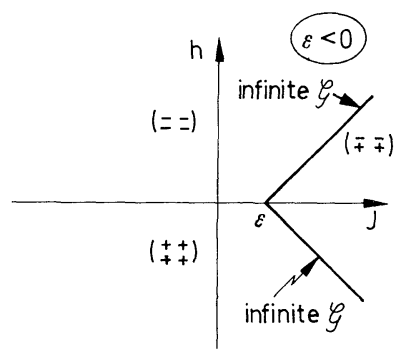

Fig. 4 
Peierls condition, and the closure condition, is satisfied everywhere except on the inclined lines, and on these lines the number of ground states is infinite.

If $v \geqq 3$, because of presence of local ground states

$$
\left(\begin{array}{cc}
+ & + \\
- & -
\end{array}\right) \text { etc. }
$$

one has to choose different decomposition of $H$. Presumably cubes as elementary domains would do. But this leads to much more computations than in the twodimensional case; we did not try it.

\section{Spin Version of Eight Vertex Model [12]}

Let

$$
\Phi_{0}=J \sigma_{a} \sigma_{c}+J^{\prime} \sigma_{b} \sigma_{d}+J_{4} \sigma_{a} \sigma_{b} \sigma_{c} \sigma_{d}
$$

and

$$
H=-\sum_{x \in \mathbb{Z}^{2}} \Phi_{0} \circ \tau_{x} .
$$

The interaction is invariant under flipping of all the spins on $\mathbb{L}_{0}$ and $\mathbb{L}_{1}$ separately (cf. Sections 5 and 6 for notation). Hence there are at least four ground states if there is one. For $J_{4}=0$ one obtains two independent Ising models, and the case of $J_{4}<0$ is related to that of $J_{4}>0$ by a simple transformation [12]. Therefore we consider the later case only. Proceeding as before in applying Criterium we obtain the phase diagram of [12, Fig. 2] which was deduced from Baxter's solution of Eight Vertex Model:

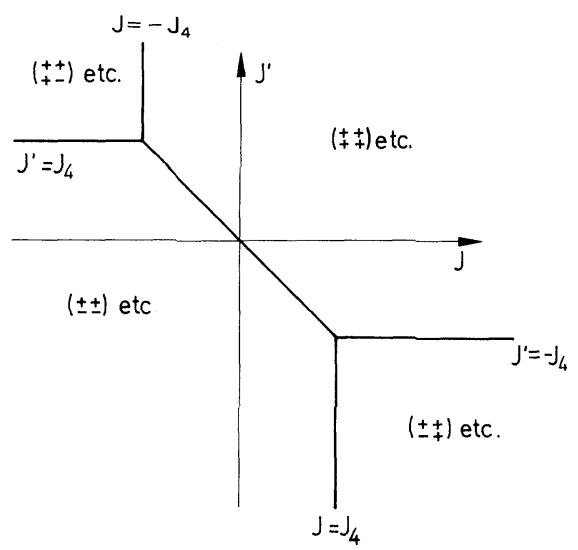

Fig. 5

here etc. stays for three ground states obtained from the one indicated on the diagram by applying the symmetry operations; Peierls condition is satisfied everywhere except on the lines of the diagram and on these lines the number of ground states is infinite. 


\section{Spin1/2 Ferromagnetic Systems}

$S=\{-1,1\}$ (as before),

$$
\Phi_{\Lambda}=-J(\Lambda) \sigma_{\Lambda},
$$

where

$$
\sigma_{\Lambda}=\prod_{a \in \Lambda} \sigma_{a}
$$

$\Lambda \mapsto J(\Lambda)$ is periodic, non-negative, and $J(\Lambda)=0$ if the diameter of $\Lambda$ is large enough; the last condition, finite range of the interaction, is unessential.

Let

$$
\begin{aligned}
\mathscr{B} & =\{\Lambda: J(\Lambda) \neq 0\} \\
\mathscr{S} & =\left\{X \in \mathscr{X}: \sigma_{B}(x)=1, \quad \forall B \in \mathscr{B}\right\} .
\end{aligned}
$$

By Lemma $2, \mathscr{G}=\mathscr{S}^{\text {per }}$ (the family of all periodic elements of $\mathscr{S}$ ).

By Appendix if $\mathscr{S}$ is infinite then so is $\mathscr{S}^{\text {per }}$. In case $\mathscr{S}$ is finite it follows from Criterium that $\mathscr{S}=\mathscr{G}$ and Peierls condition holds. Thus ferromagnetic $\operatorname{spin}_{2}^{1}$ system satisfies Peierls condition if and only of $\mathscr{S}$ is finite.

This allows us, in case of a finite $\mathscr{S}$, to deduce the results of [7] from that of [4] or [9]. Since $\mathscr{S}$ may be infinite in general, it is of interest to have a simple criterium for its finitness; this is discussed in Section 12.

\section{Generalized Ferromagnetic Systems}

These include higher spin ferromagnetic systems; cf. [11] for more details.

$S$ is a finite set of real numbers, invariant with respect to $s \mapsto-s$.

A multiplicity function (m.f.) is a map $\mathbb{L} \rightarrow\{0,1,2, \ldots\}$, zero almost everywhere. For a m.f. $B$ let

$$
s_{B}(x)=\prod_{a \in \mathbb{L}}\left(X_{a}\right)^{B(a)} .
$$

A ferromagnetic interaction is of the form

$$
H(X \mid Y)=-\sum_{B \in \mathscr{B}} J(B)\left(s_{B}(X)-s_{B}(Y)\right)
$$

with $J(B)>0$ for all $B \in \mathscr{B}$, where $\mathscr{B}$ is a periodic family of m.f.'s, $J$ is periodic and we assume that for any $a \in \mathbb{L}$

$$
\{B \in \mathscr{B}: B(a) \neq 0\}
$$

is finite ("finite range"), and non-empty. If $m=\sup \{s: s \in S\}$ then the configuration E,

$$
E_{a}=m, \quad \forall a \in \mathbb{L},
$$

is a ground state and for any ground state $X$ :

$$
\left|X_{a}\right|=m, \quad \forall a \in \mathbb{L} ;
$$

this follows from Lemma 2. 
Let the group $\{-1,1\}^{\mathbb{L}}$ act on $\mathscr{X}$ by

$(G \cdot X)_{a}=G_{a} X_{a}, \quad G=\left(G_{a}\right)_{a \in \mathbb{L}}, G_{a}= \pm 1$.

Then for any m.f. $A$

$$
s_{A} \circ G=\sigma_{\bar{A}}(G) s_{A}
$$

with

$$
\bar{A}=\{a \in \mathbb{L}: A(a) \text { is an odd number }\} .
$$

Defining the symmetry group $\mathscr{S}$ by

$$
\mathscr{S}=\left\{G \in\{-1,1\}^{\mathbb{L}}: \sigma_{\bar{B}}(G)=1, \forall B \in \mathscr{B}\right\}
$$

we see that the map $G \mapsto G \cdot E$ sets one-to-one correspondence between $\mathscr{S}^{\text {per }}$ and $\mathscr{G}(H)$. Applying Criterium and the result of Appendix we obtain that the system satisfies Peierls conditions if and only if $\mathscr{S}$ is finite.

\section{Finitness of the Number of Ground States in Two Dimensions}

The framework is as in Section 10 with additional assumption that $\mathscr{B}$ is $\mathbb{Z}^{\nu}$-invariant. We refer to [7] for the notion of reduction, for notation and for the algebra; see also $[6,11]$. We recall only that any system has a reduced version with the same number of extremal periodic equilibrium states as the original one. The system is reduced if the greatest common divisor of $\mathscr{B}$ is trivial (g.c.d. $\mathscr{B}=1$ ), and to verify the last condition one has to find the greatest common divisor of a family of polynomials in $v$ variables associated with the system. Our result is that reduced systems in two dimensions have finite number of ground states:

Theorem. In two dimensions, if g.c.d. $\mathscr{B}=1$ then $\mathscr{S}$ is finite.

(If g.c.d. $\mathscr{B}$ is non-trivial then $\mathscr{S}$ is infinite.) The theorem is easily deduced from the following.

Lemma. If $\mathscr{B}$ is $\mathbb{Z}^{v}$-invariant and g.c.d. $\mathscr{B}=1$ then for any $i=1, \ldots, v, \overline{\mathscr{B}}$ contains a non-empty subset of the hyperplane $\left\{x \in \mathbb{Z}^{v}: x_{i}=0\right\}$.

For if $v=2$ and $\overline{\mathscr{B}}$ contains non-empty subsets $A, B, A \subset \mathbb{Z} \cdot e_{1}, B \subset \mathbb{Z} \cdot e_{2}$ then elements of $\mathscr{S}$ are uniquely determined by their restrictions to any rectangle with sides of length $\operatorname{diam}(A)$ and $\operatorname{diam}(B)$. Hence $\mathscr{S}$ has no more than

$2^{\operatorname{diam}(A) \times \operatorname{diam}(B)}$

elements.

To prove the lemma it is enough to show that

if $P_{1}, \ldots, P_{n} \in \mathbb{F}_{2}\left[X_{1}, \ldots, X_{v}\right]$ are relatively prime then there exist $Q_{1}, \ldots, Q_{n} \in \mathbb{F}_{2}\left[X_{1}, \ldots, X_{v}\right]$ such that $Q_{1} P_{1}+\ldots+Q_{n} P_{n}$ is in $\mathbb{F}_{2}\left[X_{2}, \ldots, X_{v}\right]$.

For a proof identify $\mathbb{F}_{2}\left[X_{1}, \ldots, X_{v}\right]$ with $\mathbb{A}\left[X_{1}\right]$ where $\mathbb{A}=\mathbb{F}_{2}\left[X_{2}, \ldots, X_{v}\right]$. By assumption, $P_{1}, \ldots, P_{n}$ are relatively prime elements of $\mathbb{A}\left[X_{1}\right]$. By $[1], P_{1}, \ldots, P_{n}$ are relatively prime also when considered as elements of $\mathbb{K}\left[X_{1}\right]$ where $\mathbb{K}$ is the 
field of fractions of $\mathbb{A}$. Hence there exist $\tilde{Q}_{1}, \ldots, \tilde{Q}_{n} \in \mathbb{K}\left[X_{1}\right]$ such that

$$
\tilde{Q}_{1} \cdot P_{1}+\ldots+\tilde{Q}_{n} P_{n}=1 .
$$

Since $\mathbb{K}$ is the field of fractions of $\mathbb{A}$ there exists $R \in \mathbb{A}$ such that $R \cdot Q_{i} \in \mathbb{A}\left[X_{1}\right]$ $i=1, \ldots, n$. Setting $Q=R \cdot \tilde{Q}_{i}$ we see that

$$
Q_{1} \cdot P_{1}+\ldots+Q_{n} P_{n}=R
$$

is in $\mathbb{A}$. The theorem is proved.

In case of generalized ferromagnetic systems, a $\mathbb{Z}^{v}$-invariant system need not be equivalent to a reduced one. But the theorem of this section applied to $\{\bar{B}: B \in \mathscr{B}\}$ instead of $\mathscr{B}$ yields a criterium of finitness of the number of ground states. In case of spin systems, even if g.c.d. $\{\bar{B}: B \in \mathscr{B}\}$ is non-trivial, and therefore the number of ground states is infinite, the theorem and the results of [11] show that, nevertheless, there is only a finite number of extremal periodic equilibrium states at low temperatures.

\section{Systems with Constrains}

We do not discuss the most general type of constrains for which Lemma of Section 2 holds. We note only the following sufficient condition ("specification"): there exists $\delta>0$ such that if $\Lambda \subset \mathbb{L}, \Lambda^{\prime} \subset \mathbb{L}$, $\operatorname{dist}\left(\Lambda, \Lambda^{\prime}\right) \geqq \delta$ and $X \in \mathscr{X}_{\Lambda}, X^{\prime} \in \mathscr{X}_{\Lambda^{\prime}}$, then there exists $Y \in \mathscr{X}_{\Lambda \cup A^{\prime}}$ with $\operatorname{pr}_{\Lambda} Y=X, \operatorname{pr}_{A^{\prime}} Y=X^{\prime}$.

Suppose we start with a system with no constrains $\left(S^{\mathbb{L}}\right.$ as the configuration space) and with an interaction $\Psi$ to which Criterium applies yielding a finite family $\mathscr{G}(\Phi)$ of ground states. Suppose $\mathscr{X} \subset S^{\mathbb{L}}$ is the configuration space of a new system whose interaction $\Phi$ is the restriction of $\Psi$ to $\mathscr{X}$. If $\mathscr{X}$ satisfies the condition above and $\mathscr{X} \cap \mathscr{G}(\Psi)$ is non-empty then it follows from Criterium that $\mathscr{G}(\Phi)=\mathscr{X} \cap \mathscr{G}(\Psi)$ and $\Phi$ satisfies Peierls condition.

For example, let

$$
\mathscr{X}=\left\{X \in\{-1,1\}^{2}: \text { if } a, b \in \mathbb{Z}^{2} \text { are } n . n \text { then } X_{a} \text { or } X_{b}=-1\right\} \text {. }
$$

$\mathscr{X}$ satisfies the condition above since if $\operatorname{dist}\left(\Lambda, \Lambda^{\prime}\right)>1, X \in \mathscr{X}_{\Lambda}, X^{\prime} \in \mathscr{X}_{\Lambda}$, then one can define $Y$ by:

$$
Y \uparrow \Lambda=X, \quad Y \uparrow \Lambda^{\prime}=X^{\prime}, \quad Y_{a}=-1, \quad \forall a \in \mathbb{Z}^{2} \backslash\left(\Lambda \cup \Lambda^{\prime}\right) .
$$

$\mathscr{X}$ is the configuration space of the model considered in [2]. The remark of the preceding paragraph allows us to deduce the results of [2] from those of Pirogov and Sinai.

In case $\mathscr{G}(\Psi) \cap \mathscr{X}=\emptyset$ constrains may lead to drastic changes of the phase diagram: with same as above configuration space, consider the Ising model in magnetic field,

$$
H=-2 \sum_{n . n} \sigma_{a} \sigma_{b}+4 h \sum_{a} \sigma_{a} .
$$

Defining

$$
\Psi_{0}=-\left(\sigma_{a} \sigma_{b}+\sigma_{b} \sigma_{c}+\sigma_{c} \sigma_{d}+\sigma_{d} \sigma_{a}\right)+h\left(\sigma_{a}+\sigma_{b}+\sigma_{c}+\sigma_{d}\right)
$$




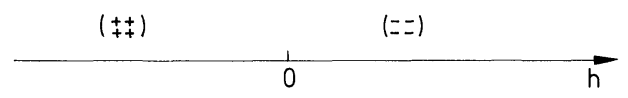

Fig. 6

(cf. Section 5 for notation) and applying Criterium the following is obtained. With $\{-1,1\} \mathbb{Z}^{2}$ as the configuration space, the phase diagram at $T=0$ is as on Figure 6, i.e. one ground state for $h>0$, one for $h<0$, and two for $h=0$. Peierls condition holds for all values of $h$.

With $\mathscr{X}$ as the configuration space, $\mathscr{G}(\Psi) \cap \mathscr{X}=\emptyset$ for $h<0$. Hence configurations different from $\left(\begin{array}{ll}+ & + \\ + & +\end{array}\right)$ enter the competition. One obtains the following local ground states in $\Lambda_{0}$ :

$$
( \pm \mp)(\mp \pm)
$$

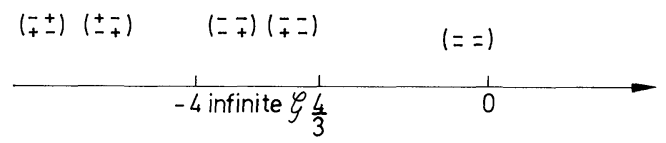

Fig. 7

Peierls condition holds for $h>-\frac{4}{3}$ and $h<-4$. It does not hold for $-4 \leqq h \leqq-\frac{4}{3}$ since, as it is not hard to see, in this region the four local ground states combine into infinitely many ones.

Acknowledgements. J.S. would like to thank the Centre de Physique Théorique, CNRS, Marseille, Mathematics Department, The University of Western Ontario, and the Institut de Physique Théorique, Universite de Louvain for their kind hospitality extended during the time this work was performed.

\section{Appendix}

We show here that if in a ferromagnetic system the group $\mathscr{S}$ is infinite then so is the subgroup $\mathscr{S}^{\text {per }}$ of its periodic elements; this result is used in Section 10. In case translations act transitively on the lattice, we prove a stronger statement: $\mathscr{S}^{\text {per }}$ is dense in $\mathscr{S}$.

The notation is as in Section 3 and in Appendix B of [7].

1. $\mathscr{X}=\{-1,1\}^{\mathbb{Z}^{v}}$; the dual group $\hat{\mathscr{X}}$ of $\mathscr{X}$ is identified with (the additive group of) $\mathbb{F}_{2}\left[\mathbb{Z}^{v}\right]\left(=\mathscr{P}_{f}\left(\mathbb{Z}^{v}\right)\right)$ equipped with the discrete topology. In particular we can speak about orthogonality of elements of $\mathscr{X}$ and $\mathbb{F}_{2}\left[\mathbb{Z}^{\nu}\right]$.

It is readily seen from [7] that if $\mathbb{G}$ is a subgroup of $\mathbb{Z}^{v}$ with generators $a_{1}, \ldots, a_{v}$ then an element of $\mathscr{X}$ is $\mathbb{G}$-invariant if and only if it is orthogonal to

$$
\left(\mathbb{1}+X^{a_{1}}, \ldots, \mathbb{1}+X^{a_{v}}\right),
$$

the ideal of $\mathbb{F}_{2}\left[\mathbb{Z}^{v}\right]$-generated by $\mathbb{1}+X^{a_{1}}, \ldots, \mathbb{1}+X^{a_{v}}$.

Hence if $\mathscr{S}$ is a closed translation invariant subgroup of $\mathscr{X}$ and $\mathscr{I}=\mathscr{S}^{\perp}$ is the corresponding ideal of $\mathbb{F}_{2}\left[\mathbb{Z}^{v}\right]$ the set of $\mathbb{G}$-invariant elements of $\mathscr{S}$ coincides with

$$
\left(\mathscr{I}, \mathbb{1}+X^{a_{1}}, \ldots, \mathbb{1}+X^{a_{v}}\right)^{\perp} .
$$

It follows that the set $\mathscr{S}^{\text {per }}$ of periodic elements of $\mathscr{S}$ is

$$
\bigcup_{n_{1}, \ldots, n_{v} \in \mathbb{N}}\left(\mathscr{I}, \mathbb{1}+X_{1}^{n_{1}}, \ldots, \mathbb{1}+X_{v}^{n_{v}}\right)^{\perp}
$$


$\mathbb{N}=\{1,2,3, \ldots\}$. And since $\mathscr{S}^{\text {per }}$ is a subgroup of $\mathscr{S}, \mathscr{S}^{\text {per }}$ is dense in $\mathscr{S}$ if and only if and anby if

$$
\left(\bigcup_{n_{1}, \ldots, n_{v} \in \mathbb{N}}\left(\mathscr{I}, \mathbb{1}+X_{1}^{n_{1}}, \ldots, \mathbb{1}+X_{v}^{n_{v}}\right)\right)^{\perp}=\mathscr{I} .
$$

The left hand side here is equal to

$$
\bigcap_{n_{1}, \ldots, n_{v} \in \mathbb{N}}\left(\mathscr{I}, \mathbb{1}+X_{1}^{n_{1}}, \ldots, \mathbb{1}+X_{v}^{n_{v}}\right) .
$$

Therefore the density of $\mathscr{S}^{\text {per }}$ in $\mathscr{S}$ follows from the following:

for any different from $\mathbb{1}$ invertible element $X$ of $\mathbb{F}_{2}\left[\mathbb{Z}^{v}\right]$ and for any ideal of $\mathrm{F}_{2}\left[\mathbb{Z}^{v}\right]$

$$
\bigcap_{n \in \mathbb{N}}\left(\mathscr{I}, \mathbb{1}+X^{n}\right)=\mathscr{I} .
$$

Barry Simon has kindly passed the above to Prof. N. Katz of Princeton University who provided a proof of it; we do not reproduce the proof here.

2. In the general case, let

$$
\mathbb{L}=\mathbb{L}_{1} \cup \ldots \cup \mathbb{L}_{n}
$$

be the decomposition of $\mathbb{L}$ into different $\mathbb{Z}^{v}$-orbits. We have:

$$
\mathscr{X}=\mathscr{X}_{1} \times \ldots \times \mathscr{X}_{n},
$$

where

$$
\mathscr{X}_{i}=\{-1,1\}^{\mathbb{L}_{i}},
$$

and

$$
\mathscr{P}_{f}(\mathbb{L})=\mathscr{P}_{f}\left(\mathbb{L}_{1}\right) \oplus \ldots \oplus \mathscr{P}_{f}\left(\mathbb{L}_{n}\right)
$$

each of $\mathscr{P}_{f}\left(\mathbb{L}_{i}\right)$ being isomorphic, as a $\mathbb{F}_{2}\left[\mathbb{Z}^{v}\right]$-module, to $\mathbb{F}_{2}\left[\mathbb{Z}^{v}\right]$. Let $p_{i}, i=1, \ldots, n$, stand for the projections $\mathscr{X} \rightarrow \mathscr{X}_{i}, \mathscr{P}_{f}(\mathbb{L}) \rightarrow \mathscr{P}_{f}\left(\mathbb{L}_{i}\right)$ and $p_{i}$ for the complementary projection $\mathscr{X} \rightarrow \mathscr{X} \times \ldots \times \mathscr{X}_{i-1} \times \mathscr{X}_{i+1} \times \ldots \times \mathscr{X}_{n}$.

By induction with respect to the number $n$ of $\mathbb{Z}^{v}$-orbits we will prove that for any infinite, closed, $\mathbb{Z}^{v}$-invariant subgroup $\mathscr{S}$ of $\mathscr{X}, \mathscr{S}^{\text {per }}$ is infinite. The case $n=1$ is already dealt with.

Since $\mathscr{S}$ is infinite, one of the projections $p_{i}(\mathscr{S}), i=1, \ldots, n$, is infinite; we may assume that $p_{1}(\mathscr{S})$ is infinite. If $\mathscr{S} \cap\left(\mathscr{X}_{2} \times \ldots \times \mathscr{X}_{n}\right)$ is infinite then, by the inductive assumption $\left(\mathscr{S} \cap\left(\mathscr{X}_{2} \times \ldots \times \mathscr{X}_{n}\right)\right)^{\text {per }} \subset \mathscr{S}^{\text {per }}$ is infinite too; here $\mathscr{X}_{2} \times \ldots \times \mathscr{X}_{n}$ is identified with the subgroup $\{0\} \times \mathscr{X}_{2} \times \ldots \times \mathscr{X}_{n}$ of $\mathscr{X}$. Hence we may assume that $\mathscr{S}_{1}=\mathscr{S} \cap\left(\mathscr{X}_{2} \times \ldots \times \mathscr{X}_{n}\right)$ is finite.

Since $p_{1}(\mathscr{S})$ is a closed infinite $\mathbb{Z}^{v}$-invariant subgroup of $\mathscr{X}_{1}$ periodic elements are dense in it. In particular it contains an infinite number of periodic elements. It is therefore enough to show that for any periodic $X \in p_{1}(\mathscr{S})$ there is a periodic $Y \in p_{1},(\mathscr{S})$ such that $X+Y \in \mathscr{S}$.

Thus, let $X$ be an element of $p_{1}(\mathscr{S})$ which is invariant under a cofinite subgroup $\mathbb{G}$ of $\mathbb{Z}^{v}$; let $Y \in p_{1},(\mathscr{S})$ be such that $X+Y \in \mathscr{S}$. Then for any $g \in \mathbb{G}$

$$
Z(g)=g \cdot Y-Y
$$


is in $\mathscr{S}_{1}$, since $Z(g) \in \mathscr{X}_{2} \times \ldots \times \mathscr{X}_{n}$ and

$$
Z(g)=g \cdot(X+Y)-(X+Y) \in \mathscr{S} .
$$

By direct calculation:

$$
Z\left(g_{1}+g_{2}\right)=g_{1} Z\left(g_{2}\right)+Z\left(g_{1}\right), \quad Z(0)=0
$$

(i.e. $Z$ is a $\mathscr{S}_{1}$-valued cocycle). Since $\mathscr{S}_{1}$, is finite it is pointwise invariant under a cofinite subgroup, say $\mathbb{G}^{\prime}$, of $\mathbb{Z}^{v}$. Hence the above identities show that $Z$ is a homomorphism $\mathbb{H} \rightarrow \mathscr{S}_{1}$, where $\mathbb{H}=\mathbb{G} \cap \mathbb{G}^{\prime}$ is again a cofinite subgroup of $\mathbb{Z}^{v}$. Since all elements of $\mathscr{X}$ are of order 2 it follows that $Z(2 g)=0$ for any $g \in \mathbb{H}$. Thus $Y$ is invariant under the cofinite subgroup $2 \cdot \mathbb{H}$ of $\mathbb{Z}^{\nu}$. This ends the proof.

\section{References}

1. Bourbaki,N.: Algèbre commutative, Chapter 7 (§3, Theorem 2). Paris: Hermann 1965

2. Cassandro,M., Da Fano, A., Olivieri,E.: Commun. math. Phys. 44, 45-51 (1975)

3. Dobrushin, R. L.: Funct. Anal. Applic. 2, 302-312 (1968)

4. Gertsik,V.M.: Izv. Akad. Nauk. SSR, Ser. Mat. 40, 448-462 (1976)

5. Gertsik,V.M., Dobrushin, R.L.: Funkts. Analiz. 8, 12 (1974)

6. Holsztynski,W., Slawny,J.: Lett. Nuovo Cimento 13, 534-538 (1975)

7. Holsztynski,W., Slawny,J.: Phase transitions in ferromagnetic spin systems at low temperatures. Preprint (1975)

8. Pirogov, S. A.: Izv. Akad. Nauk SSR, Ser. Mat. 39, 1404-1442 (1975)

9. Pirogov, S. A., Sinai, Ya.G.: Teor. Mat. Fiz. 25, 358-369 (1975); 26, 61-76 (1976)

10. Ruelle,D.: Statistical mechanics. Rigorous results. New York: Benjamin 1969

11. Slawny,J.: Commun. math. Phys. 46, 75-97 (1976)

12. Wu, F. Y.: Phys. Rev. B 4, 2312-2314 (1971)

13. Griffiths, R. B.: Phys. Rev. B 7, 545-551 (1973)

14. Ruelle,D.: Formalisme thermodynamique. In: Séminaire Bourbaki 1974/75. Lecture notes in mathematics, Vol. 514. Berlin-Heidelberg-New York: Springer 1976

Communicated by E. Lieb

Received December 16, 1977 